\title{
Relation between Carotid Stenosis Severity, Plaque Echogenicity Characteristics and IMT Assessed by Ultrasound in the Community Population of Southern China
}

\author{
Huanquan Liao ${ }^{*}$, Hua Hong1*\#, Hongxuan Wang2 \\ ${ }^{1}$ Department of Neurology, The First Affiliated Hospital, Sun Yat-Sen University, Guangzhou, China \\ ${ }^{2}$ Department of Neurology, The Second Affiliated Hospital, Sun Yat-Sen University, Guangzhou, China \\ Email: "sumsdiy@163.com
}

Received 24 September 2015; accepted 18 October 2015; published 23 October 2015

Copyright (C) 2015 by authors and OALib.

This work is licensed under the Creative Commons Attribution International License (CC BY). http://creativecommons.org/licenses/by/4.0/

c) $\underset{\mathrm{EY}}{\mathrm{i}}$ Open Access

\begin{abstract}
Carotid artery atherosclerosis (CAA) is one of the major high-risk mechanisms of stroke. Relationship between IMT, carotid plaque echogenicity and carotid stenosis assessed by ultrasound has not been systemically studied, especially in subjects with mild to moderate carotid stenosis. In this study we measured these 3 different ultrasound-derived quantitative traits concurrently in the community population of southern China and compared their associations. We found that IM$\mathrm{T}_{\text {mean }}$ in the non-stenosis group $(0 \%),<30 \%$ group and $\geq 30 \%$ group were $0.72 \pm 0.11 \mathrm{~mm}, 0.85 \pm$ $0.13 \mathrm{~mm}, 0.90 \pm 0.17 \mathrm{~mm}$ respectively. $\mathrm{IMT}_{\text {mean }}$ of the $<30 \%$ group and $\geq 30 \%$ group were higher than that of $0 \%$ group $(P<0.01), \geq 30 \%$ group was higher than $<30 \%$ group $(P<0.01)$. IMT $_{\text {mean }}$ in the no-plaque group, hyperechoic group and hypoechoic group were $0.75 \pm 0.13 \mathrm{~mm}, 0.87 \pm 0.17$ $\mathrm{mm}, 0.84 \pm 0.15 \mathrm{~mm}$ respectively. IMT $_{\text {mean }}$ of the hyperechoic group and the hypoechoic group was

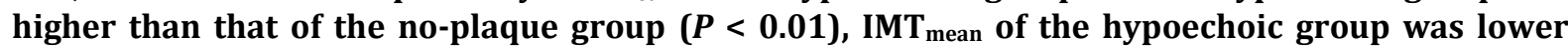
than that of the hyperechoic group, though with no statistic significance $(P=0.58)$. The proportion of hyperechoic or hypoechoic plaque in the $<30 \%$ group was higher than that in the non-stenosis group $(P<0.01)$, as well as proportion of hypoechoic plaque in the $\geq 30 \%$ group was higher than that in the $<30 \%$ group $(P<0.01)$. Our study indicated that there is definite correlation between stenosis severity, plaque echogenicity characteristics and IMT in evaluation the extent of CAA with ultrasound. The result suggests that the 3 different ultrasound-derived quantitative traits, are a progressing process, and this process increases the risk of stroke, thus medical treatment especially stabilizing the plaques is necessary.
\end{abstract}

\footnotetext{
*These authors contributed equally to this work.

${ }^{\#}$ Corresponding author.
}

How to cite this paper: Liao, H.Q., Hong, H. and Wang, H.X. (2015) Relation between Carotid Stenosis Severity, Plaque Echogenicity Characteristics and IMT Assessed by Ultrasound in the Community Population of Southern China. Open Access Library Journal, 2: e2014. http://dx.doi.org/10.4236/oalib.1102014 


\title{
Keywords
}

\section{Ultrasound, Carotid Stenosis, Plaque Characteristic, Intima-Media Thickness}

\author{
Subject Areas: Neuroscience
}

\section{Introduction}

Stroke remains one of the most devastating neurological diseases, often causing death, or gross physical impairment [1]. Carotid artery atherosclerosis (CAA) is one of the major high-risk mechanisms of stroke [2]. At present, degree of stenosis and symptomatology are the main grounds to perform carotid endarterectomy (CEA). Pooled analysis of large randomized controlled trials showed that CEA is highly beneficial for symptomatic patients with high-grade (>70\%) stenosis, reducing the 5-year absolute risk of ipsilateral ischemic stroke with 16\% [3]. However, in patients with moderate (50\% to 69\%) carotid stenosis, the 5-year absolute risk reduction of ipsilateral stroke is only $4.6 \%$, whereas CEA has no effect in symptomatic patients with mild (30\% to $49 \%$ ) carotid stenosis [3]. Studies of atherosclerosis encompass a broad range of phenotypes, including clinical events such as stroke or myocardial infarction, transient ischemic attacks or unstable coronary syndromes, and measurements derived from noninvasive assays with the use of lumenography, ultrasound, CT, or MRI. Noninvasive modalities can also measure different aspects of atherogenesis. For instance, ultrasound examination of the carotid arteries can provide determinations of intima-media thickness (IMT), echogenicity of carotid plaques, or severity of arterial stenosis. Although these phenotypes each assay "atherosclerosis", they represent different stages of atherogenesis, which is a complex multistep process that has many physical, biochemical, molecular, and genetic determinants [4]. However, the relationship between these phenotypes assessed by ultrasound has not been systemically studied, especially in subjects with mild to moderate carotid stenosis. In this study we compared the association between 3 different ultrasound-derived quantitative traits, namely, IMT, carotid plaque echogenicity and carotid stenosis, measured concurrently in the community population of southern China, which were the same group of individuals.

\section{Methods}

\subsection{Study Cohort}

Study participants were enrolled from community populations in Guangzhou, China. Participants were enrolled by the Department of Neurology, the First Affiliated Hospital of Sun Yat-sen University from July 2008 to December 2008. Those who are aged from 46 to 75 with Chinese ethnic were eligible for the study. Those who had malignant tumors, acute or sub-acute symptomatic cardiovascular diseases (the period between the recovery from the diseases and the study recruitment was less than six months), and other critical illnesses were excluded because a potential possibility to be lost to follow-up. Those who had cardiovascular diseases more than six months ago and totally recovered without any persistent symptoms, sequelae or disabilities were not excluded in the study. However, People who refused to complete necessary questionnaires were also excluded. Among 1025 residents who participated in our study, 962 residents completed all examinations and were included in the analysis. The study protocol was approved by the Ethics Committee of the First Affiliated Hospital of Sun Yat-sen University in May 2008, and all participants agreed on the written informed consent of the study.

\subsection{Ultrasound Measurements of Carotid IMT and Plaques}

Carotid ultrasound measurements were performed with two B-mode ultrasound systems; APLIO XU equipped with a $7.5 \mathrm{MHz}$ linear array transducer (Toshiba, USA) and HDI 5000 with a 5 - $12 \mathrm{MHz}$ linear array transducer (Philips, USA). Subjects were examined in supine position with their necks extended. Measurements were taken in the diastolic phase in a proper direction for best visualization of the arteries. IMT was measured as the distance between the two parallel echogenic lines on the far wall of artery in longitudinal plane image frozen in the screen by electronic calipers [5]. IMT in three defined locations were measured bilaterally: common carotid (20 $\mathrm{mm}$ proximal to the bifurcation), carotid bifurcation and internal carotid (10 $\mathrm{mm}$ distal to bifurcation). Mean 
IMT was calculated as the average of the six readings of bilateral carotid arteries. Plaque was defined as localized thickening of IMT $\geq 1.3 \mathrm{~mm}$ which did not uniformly involve the whole wall of carotid artery [6] [7]. Classification of ultrasound plaque appearance was performed independently from the videotape by 1 single operator blinded to all other data. 3 categories were classified as 1) no plaques; 2) hyperechoic plaques; 3) hypoechoic plaques. The carotid stenosis measurements were performed according to internationally recognized guidelines [8]. A blood flow velocity $>1.2 \mathrm{~m} / \mathrm{s}$ was used to define a stenosis with $>30 \%$ lumen diameter reduction [9]. Quality controls were made by repeated scans on several randomly selected participants who were examined twice by two sonographers. The coefficient of variance (CV) of the mean IMT was $10.3 \%$. The interobserver difference was $0.08 \pm 0.08$ and the correlation of two readings was $0.661(P<0.01)$. The agreement of plaque occurrence was $88.1 \%$ and the kappa value was $0.738(P<0.01)$.

\subsection{Statistical Methods}

Analysis of normality of the continuous variables was performed with the Kolmogorov-Smirnov test. Results for continuous variables normally distributed were expressed as mean value \pm standard deviation (SD). Dichotomized or categorized variables were described as numbers and proportions. In univariate analysis, continuous variables normally distributed were compared by student's t test. Dichotomized or categorized variables were compared by Chi-square tests. All statistic analysis was calculated with SPSS 13.0 software system (SPSS Inc., Chicago, IL, USA). A two-sided $P$-value of less than 0.05 was considered as statistically significant.

\section{Results}

\subsection{IMT Comparison between Groups Stratified by Stenosis Severity}

Figure 1 shows that $\mathrm{IMT}_{\text {mean }}$ in the non-stenosis group (0\%), $<30 \%$ group and $\geq 30 \%$ group were $0.72 \pm 0.11$ $\mathrm{mm}, 0.85 \pm 0.13 \mathrm{~mm}, 0.90 \pm 0.17 \mathrm{~mm}$ respectively. $\mathrm{IMT}_{\text {mean }}$ of the $<30 \%$ group and $\geq 30 \%$ group were higher than that of $0 \%$ group $(P<0.01), \geq 30 \%$ group was higher than $<30 \%$ group $(P<0.01)$.

\subsection{IMT Comparison between Groups Stratified by Plaque Echogenicity}

Figure 2 shows $\mathrm{IMT}_{\text {mean }}$ in the no-plaque group, hyperechoic group and hypoechoic group were $0.75 \pm 0.13 \mathrm{~mm}$, $0.87 \pm 0.17 \mathrm{~mm}, 0.84 \pm 0.15 \mathrm{~mm}$ respectively. $\mathrm{IMT}_{\text {mean }}$ of the hyperechoic group and the hypoechoic group were higher than that of the no-plaque group $(P<0.01)$, $\mathrm{IMT}_{\text {mean }}$ of the hypoechoic group was lower than that of the hyperechoic group, though with no statistic significance $(P=0.58)$.

\subsection{Plaque Characteristic Comparison between Groups Stratified by Stenosis Severity}

Figure 3 shows The proportion of hyperechoic or hypoechoic plaque in the $<30 \%$ group was higher than that in the non-stenosis group ( $P<0.01$ ), as well as proportion of hypoechoic plaque in the $\geq 30 \%$ group was higher than that in the $<30 \%$ group $(P<0.01)$.

\section{Discussion}

In this community-base and large sample study, we measured concurrently 3 different ultrasound-derived quantitative traits, namely, IMT, carotid plaque echogenicity and carotid stenosis in the community population of southern China and compare the association between these characteristics.

CAA is one of the risk factors of cerebral infarction [10]. Epidemiologic estimates of first-time ischemic stroke attributable to CAA vary, but range from roughly $7 \%$ to $18 \%$ of all incident stroke [11] [12]. Carotid ultrasound examination is of great value since it is noninvasive, and can reflect the degree of CAA stenosis, plaque characteristics and IMT. On the basis of CAA, the carotid stenosis decreased distal blood flow and formation of thrombosis which is an important mechanism of cerebral infarction [13] [14]. Hypoechoic plaques are mainly composed by the deposition of cholesterol, necrosis tissue and plaque bleeding, a type of plaque with instability [15]. IMT is a main indicator of the early lesions of the arterial wall [16], and is also a risk factor for future cerebral vascular events [17]. Although previous study has reported a significant correlation between IMT and plaque formation rate [18], however, study on the correlation between the degree of CAA stenosis, the echogenicity of the plaque and the IMT is rare. 


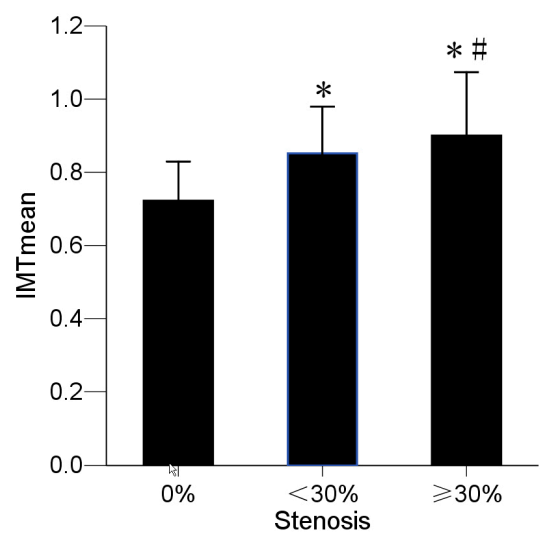

${ }^{*}$ Compared with $0 \%$ stenosis group, $P<0.01 ;{ }^{\#}$ Compared with $<30 \%$ stenosis group, $P<0.01$.

Figure 1. IMT comparison between groups stratified by stenosis severity.

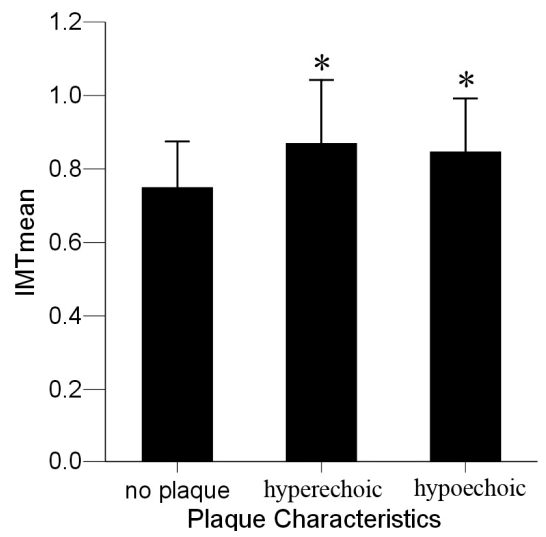

Figure 2. IMT comparison between groups stratified by echogenicity. Compared with no-plaque group, $P<0.01$.

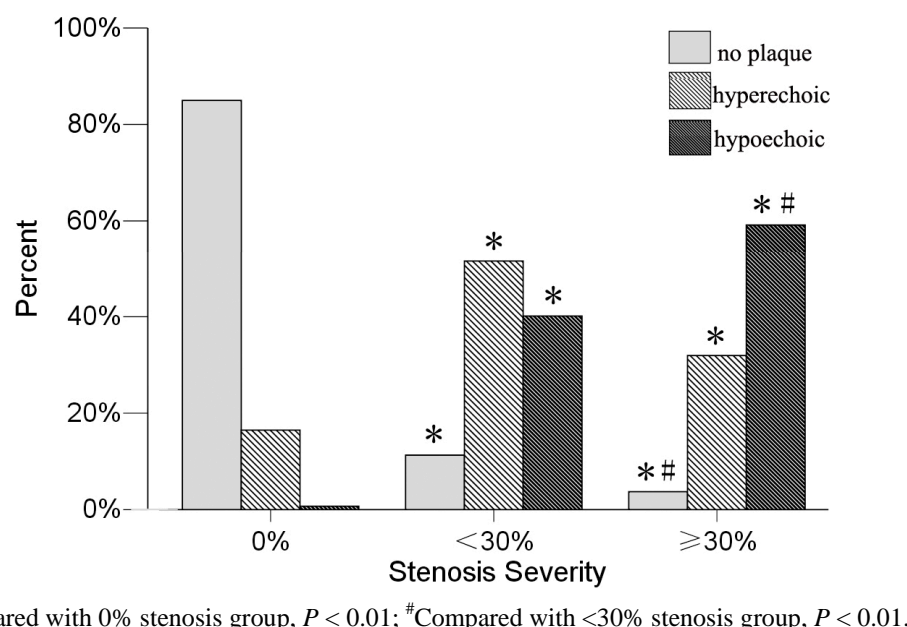

Figure 3. Plaque characteristic comparison between groups stratified by stenosis severity.

In the present study, we found that relationship between the degree of stenosis, the nature of the plaques and the the IMT were all correlated. With the increased degree of stenosis, IMT increased. IMT $_{\text {mean }}$ of the $<30 \%$ group and $\geq 30 \%$ group were higher than that of $0 \%$ group, $\geq 30 \%$ group was higher than $<30 \%$ group. On the formation of plaque, with the degree of stenosis aggravated, plaque or hypoechogenic plaque rate increased. 
However, the relation between plaque and IMT was different, although IMT in the hyperechoic plaque group was larger than that in no-plaque group, the difference in IMT between hypoechoic plaque and hyperechoic plaque groups has no statistical difference. Indeed, IMT in the hyporechoic plaque group was even smaller than that in hyperechoic plaque group. The cause of this phenomenon may be attributed to the different age distribution and was consistent with the research results of Joakimsen [19] and Zureik [20]. They explained the phenomenon due to the short life in patients with unstable plaque.

On the relationship between the degree of carotid stenosis and stroke, previous studies have focused more on severe carotid stenosis. Marquardt [21] proposed that the risk of stroke is small when asymptomatic patients with carotid stenosis more than $50 \%$ were on the premise of medical treatment. North american symptomatic carotid endarterectomy trial, european carotid surgery test and asymptomatic carotid atherosclerosis study have concluded that the severe carotid stenosis (60\% with symptoms or, higher than $70 \%$ without symptoms) patients, should be implemented with carotid endarterectomy or interventional therapy, in order to reduce the risk of ischemic stroke [22]-[24]. Our study indicated that, in the early stage prior to severe stenosis, the CAA stenosis, plaque formation and collapse are already a progressing process, and this process increases the risk of stroke. In Rothwell's study [25], angiographic plaque surface irregularity is associated with an increased risk of ipsilateral ischemic stroke on medical treatment at all degrees of stenosis. The increase in stroke risk with degree of stenosis is partly accounted for by the parallel increase in plaque surface irregularity and thrombus formation, but the degree of narrowing of the vessel lumen is still an independent predictor of ischemic stroke within 2 years of presentation. Therefore, our study indicated that in patients with mild-to-moderate carotid who are asymptomatic, medical treatment especially stabilizing the plaques is necessary.

\section{Conclusion}

In summary, there is a definite correlation between stenosis severity, plaque echogenicity and IMT in evaluation the extent of CAA with ultrasound. The result suggests that the 3 different ultrasound-derived quantitative traits are a progressing process, and this process increases the risk of stroke, thus medical treatment especially stabilizing the plaques is necessary.

\section{References}

[1] Mukherjee, D. and Patil, C.G. (2011) Epidemiology and the Global Burden of Stroke. World Neurosurgery, 76, S85S90. http://dx.doi.org/10.1016/j.wneu.2011.07.023

[2] Mathiesen, E.B. and Johnsen, S.H. (2009) Ultrasonographic Measurements of Subclinical Carotid Atherosclerosis in Prediction of Ischemic Stroke. Acta Neurologica Scandinavica, 120, 68-72. http://dx.doi.org/10.1111/j.1600-0404.2009.01210.x

[3] Rothwell, P.M. and Warlow, C.P. (1999) Prediction of Benefit from Carotid Endarterectomy in Individual Patients: A Risk-Modelling Study. European Carotid Surgery Trialists’ Collaborative Group. The Lancet, 353, 2105-2110. http://dx.doi.org/10.1016/S0140-6736(98)11415-0

[4] Hegele, R.A. (1996) The Pathogenesis of Atherosclerosis. Clinica Chimica Acta, 246, 21-38. http://dx.doi.org/10.1016/0009-8981(96)06224-9

[5] Chambless, L.E., Folsom, A.R., Clegg, L.X., Sharrett, A.R., Shahar, E., Nieto, F.J., et al. (2000) Carotid Wall Thickness is Predictive of Incident Clinical Stroke: The Atherosclerosis Risk in Communities (ARIC) Study. American Journal of Epidemiology, 151, 478-487. http://dx.doi.org/10.1093/oxfordjournals.aje.a010233

[6] Ebrahim, S., Papacosta, O., Whincup, P., Wannamethee, G., Walker, M., Nicolaides, A.N., et al. (1999) Carotid Plaque, Intima Media Thickness, Cardiovascular Risk Factors, and Prevalent Cardiovascular Disease in Men and Women: The British Regional Heart Study. Stroke, 30, 841-850. http://dx.doi.org/10.1161/01.STR.30.4.841

[7] Li, C., Engstrom, G., Berglund, G., Janzon, L. and Hedblad, B. (2008) Incidence of Ischemic Stroke in Relation to Asymptomatic Carotid Artery Atherosclerosis in Subjects with Normal Blood Pressure. A Prospective Cohort Study. Cerebrovascular Diseases, 26, 297-303. http://dx.doi.org/10.1159/000149577

[8] Zierler, R. and Strandness, D. (1987) Noninvasive Dynamic and Real-Time Assessment of Extracranial Cerebrovasculature. In: Woods, J., Ed., Cerebral Blood Flow: Physiologic and Clinical Aspects, McGraw-Hill, New York, 819-829.

[9] Zbornikova, V., Lassvik, C. and Johansson, I. (1985) Prospective Evaluation of the Accuracy of Duplex Scanning with Spectral Analysis in Carotid Artery Disease. Clinical Physiology, 5, 257-269.

[10] Hunt, K.J., Evans, G.W., Folsom, A.R., Sharrett, A.R., Chambless, L.E., Tegeler, C.H., et al. (2001) Acoustic Shadowing on B-Mode Ultrasound of the Carotid Artery Predicts Ischemic Stroke: The Atherosclerosis Risk in Communities 
(ARIC) Study. Stroke, 32, 1120-1126. http://dx.doi.org/10.1161/01.STR.32.5.1120

[11] White, H., Boden-Albala, B., Wang, C., Elkind, M.S., Rundek, T.and Wright, C.B., et al. (2005) ischemic Stroke Subtype Incidence among Whites, Blacks, and Hispanics: The Northern Manhattan Study. Circulation, 111, 1327-1331. http://dx.doi.org/10.1161/01.CIR.0000157736.19739.D0

[12] Petty, G.W., Brown, R.J., Whisnant, J.P., Sicks, J.D., O’Fallon, W.M. and Wiebers, D.O. (1999) Ischemic Stroke Subtypes: A Population-Based Study of Incidence and Risk Factors. Stroke, 30, 2513-2516. http://dx.doi.org/10.1161/01.STR.30.12.2513

[13] Grubb, R.J., Derdeyn, C.P., Fritsch, S.M., Carpenter, D.A., Yundt, K.D., Videen, T.O., et al. (1998) Importance of Hemodynamic Factors in the Prognosis of Symptomatic Carotid Occlusion. JAMA, 280, 1055-1060. http://dx.doi.org/10.1001/jama.280.12.1055

[14] Tsiskaridze, A., Devuyst, G., de Freitas, G.R., van Melle, G. and Bogousslavsky, J. (2001) Stroke with Internal Carotid Artery Stenosis. Archives of Neurology, 58, 605-609. http://dx.doi.org/10.1001/archneur.58.4.605

[15] Schulte-Altedorneburg, G., Droste, D.W., Haas, N., Kemeny, V., Nabavi, D.G., Fuzesi, L., et al. (2000) Preoperative B-mode Ultrasound Plaque Appearance Compared with Carotid Endarterectomy Specimen Histology. Acta Neurologica Scandinavica, 101, 188-194. http://dx.doi.org/10.1034/j.1600-0404.2000.101003188.x

[16] Cobble, M. and Bale, B. (2010) Carotid Intima-Media Thickness: Knowledge and Application to Everyday Practice. Postgraduate Medicine, 122, 10-18. http://dx.doi.org/10.3810/pgm.2010.01.2091

[17] Johnsen, S.H. and Mathiesen, E.B. (2009) Carotid Plaque Compared with Intima-Media Thickness as a Predictor of Coronary and Cerebrovascular Disease. Current Cardiology Reports, 11, 21-27. http://dx.doi.org/10.1007/s11886-009-0004-1

[18] Veller, M.G., Fisher, C.M., Nicolaides, A.N., Renton, S., Geroulakos, G., Stafford, N.J., et al. (1993) Measurement of the Ultrasonic Intima-Media Complex Thickness in Normal Subjects. Journal of Vascular Surgery, 17, 719-725. http://dx.doi.org/10.1016/0741-5214(93)90116-4

[19] Joakimsen, O., Bonaa, K.H., Stensland-Bugge, E. and Jacobsen, B.K. (1999) Age and Sex Differences in the Distribution and Ultrasound Morphology of Carotid Atherosclerosis: The Tromso Study. Arteriosclerosis, Thrombosis, and Vascular Biology, 19, 3007-3013. http://dx.doi.org/10.1161/01.ATV.19.12.3007

[20] Zureik, M., Bureau, J.M., Temmar, M., Adamopoulos, C., Courbon, D., Bean, K., et al. (2003) Echogenic Carotid Plaques Are Associated with Aortic Arterial Stiffness in Subjects with Subclinical Carotid Atherosclerosis. Hypertension, 41, 519-527. http://dx.doi.org/10.1161/01.HYP.0000054978.86286.92

[21] Marquardt, L., Geraghty, O.C., Mehta, Z. and Rothwell, P.M. (2010) Low Risk of Ipsilateral Stroke in Patients with Asymptomatic Carotid Stenosis on Best Medical Treatment: A Prospective, Population-Based Study. Stroke, 41, e11e17. http://dx.doi.org/10.1161/strokeaha.109.561837

[22] Barnett, H.J., Taylor, D.W., Eliasziw, M., Fox, A.J., Ferguson, G.G., Haynes, R.B., et al. (1998) Benefit of Carotid Endarterectomy in Patients with Symptomatic Moderate or Severe Stenosis. North American Symptomatic Carotid Endarterectomy Trial Collaborators. The New England Journal of Medicine, 339, 1415-1425. http://dx.doi.org/10.1056/nejm199811123392002

[23] European Carotid Surgery Trialists’ Collaborative Group (1998) Randomised Trial of Endarterectomy for Recently Symptomatic Carotid Stenosis: Final Results of the MRC European Carotid Surgery Trial (ECST). The Lancet, 351 , 1379-1387. http://dx.doi.org/10.1016/S0140-6736(97)09292-1

[24] Moore, W.S., Young, B., Baker, W.H., Robertson, J.T., Toole, J.F., Vescera, C.L., et al. (1996) Surgical Results: A Justification of the Surgeon Selection Process for the ACAS Trial. The ACAS Investigators. Journal of Vascular Surgery, 23, 323-328. http://dx.doi.org/10.1016/S0741-5214(96)70277-X

[25] Rothwell, P.M., Gibson, R. and Warlow, C.P. (2000) Interrelation between Plaque Surface Morphology and Degree of Stenosis on Carotid Angiograms and the Risk of Ischemic Stroke in Patients with Symptomatic Carotid Stenosis. On Behalf of the European Carotid Surgery Trialists’ Collaborative Group. Stroke, 31, 615-621. http://dx.doi.org/10.1161/01.str.31.3.615 